\title{
Effect of ascorbic acid on the consequences of acute alcohol consumption in humans
}

\begin{abstract}
This study examines the influence of ascorbic acid pretreatment on ethanol clearance, toxicity, and behavioral impairment after an acute dose of ethanol in humans. Ascorbic acid or a placebo was given to 20 healthy male subjects for 2 weeks before ethanol consumption. The dose of ethanol was $0.95 \mathrm{gm} / \mathrm{kg}$ body weight and was consumed during a $2 \frac{1 / 2}{2}$-hour period. Thirty minutes after ethanol consumption, motor coordination and intellectual function were assessed by Goldberg's "Finger-Finger" and "Serial Sevens" tests. In addition, color discrimination was measured with the use of the Farnsworth-Munsell 100 Hue Color Test. Hourly blood samples were taken for 10 hours after ethanol consumption to measure serum triglyceride levels, blood lactate/pyruvate ratios, and serum enzymes. Blood ethanol clearance was also determined. Ethanol consumption elevated serum triglyceride levels and blood lactate/pyruvate ratios and impaired performance of the behavioral tests but did not alter serum enzyme levels. Ascorbic acid pretreatment resulted in significant enhancement in blood ethanol clearance and an increase in serum triglyceride levels after ethanol consumption in half of the subjects. Ascorbic acid pretreatment also resulted in improved motor coordination and color discrimination after ethanol consumption in half of the subjects. Ascorbic acid pretreatment did not influence elevated blood lactate/pyruvate ratios or impaired intellectual function. (Clin Pharmacol Ther 1987;41:502-9.)
\end{abstract}

\section{Robert L. Susick, Jr., Ph.D., and Vincent G. Zannoni, Ph.D. Ann Arbor, Mich.}

The influence of ascorbic acid on the metabolism, toxicity, and behavioral impairment of alcohol has become of interest in recent years. We have previously described an in vitro ascorbic acid-dependent ethanol oxidizing system that involves the peroxidic oxidation of ethanol by catalase.' Yunice et $\mathrm{al}^{2}{ }^{2}$ demonstrated enhanced blood ethanol clearance in ascorbic acid pretreated carotid-cannulated guinea pigs infused with ethanol. Krasner et al. ${ }^{3}$ found enhanced blood ethanol clearance in healthy male volunteers after 2 weeks of pretreatment with $1.0 \mathrm{gm}$ ascorbic acid per day.

Yunice and Lindeman ${ }^{4}$ found that pretreatment with the vitamin protected against the lethality of an acute dose of ethanol in mice and chronic ethanol exposure

From the Department of Pharmacology, the University of Michigan Medical School.

Supported in part by grants 23007 from Hoffmann-La Roche, Inc., Nutley, N.J., and 5M01-RR00042 and 2 P60 AM20572 from the National Institutes of Health.

Received for publication June 3, 1986; accepted Oct. 26, 1986.

Reprint requests: Vincent G. Zannoni, Ph.D., Department of Pharmacology-MSI, the University of Michigan Medical School, Ann Arbor, MI 48109. in the rat. Susick et al. ${ }^{5}$ have demonstrated in the guinea pig that high dietary levels of ascorbic acid protect against hepatic steatosis, necrosis, and elevated levels of SGOT and SGPT caused by chronic ethanol consumption. Protection against hepatic steatosis by ascorbic acid was also found by Yunice et al. ${ }^{2}$ in guinea pigs chronically infused with ethanol. In addition, ascorbic acid has been shown to reverse the impaired swimming behavior of mice treated with an intoxicating dose of ethanol. ${ }^{6}$

This study examines the influence of ascorbic acid pretreatment on ethanol clearance, toxicity, and behavioral impairment after an acute dose of ethanol in humans. Blood ethanol clearance, serum enzymes, serum triglyceride levels, blood lactate/pyruvate ratios, and tests of motor coordination, intellectual function, and color discrimination were determined. All of these parameters have been shown to be affected by acute ethanol consumption in animals and humans. ${ }^{711}$

\section{METHODS}

Study design. Each subject was used as his own control, proceeding once through the protocol with placebo pretreatment and once with ascorbic acid pretreat- 
ment. To rule out any learning effect with the behavioral tests, half of the subjects received the placebo pretreatment first and half received vitamin $\mathrm{C}$ pretreatment first. A minimum of 4 weeks passed between test days to avoid any carryover effect. The study was conducted in a double-blind manner; neither the subject nor experimenter on the Clinical Research Unit was aware of the identity of the pretreatment regimen.

Twenty healthy, nonsmoking male adults aged 22 to 30 years obtained from the University of Michigan student population participated in the study. The health status, history, and average amount of alcohol consumption for each subject was assessed with an interview and a questionnaire. The group of students used for the study was highly selected with regard to their prior history of alcohol consumption. Subjects selected had, on average, a weekly alcohol consumption that did not exceed 3 ounces of ethanol. A complete blood chemistry and urine analysis were also performed on each subject before alcohol administration. There was no history of health problems, liver disease, or alcoholism in any of the subjects. The study protocol was approved by the University of Michigan Human Subject Review Committee and informed consent was obtained from each subject on a form approved by the Committee. All studies were performed under medical supervision at the Clinical Research Center of the University of Michigan Hospital on the test day, as well as the day before and after the test day when the baseline values were obtained.

The study consisted of a 2-week pretreatment period during which the subject consumed either $1.0 \mathrm{gm}$ timed release ascorbic acid or placebo (lactose) five times per day. The maximum, safest pharmacologic dose of the vitamin was used to determine if there was a vita$\min \mathrm{C}$ effect. No evidence has been found indicating, that a dose of $5 \mathrm{gm}$ ascorbic acid per day, in a timedrelease form, during a short period of time (2 weeks) results in any adverse reactions. In addition, no subject in the study reported any adverse symptoms while receiving either the placebo or ascorbic acid. There were also no symptoms of vitamin $C$ deficiency in any of the subjects on cessation of ascorbic acid treatment. The subjects recorded each dose that was taken on a data card and this record was collected weekly. Subjects were also given a bottle containing a known number of pills that was collected at the end of each week. During the 2-week pretreatment period, each subject refrained from alcohol, drugs of any kind, and foods high in vitamin $C$ such as citrus fruits and juices and certain vegetables. With regard to their diet, the subjects were given an extensive list of foods to avoid prepared by the Clinical Research Center Dietetic Department. The subjects were also specifically told during the extensive interview not to consume alcohol or any drugs during the course of the study. The 2-week pretreatment period was followed by the test day at which time ethanol consumption occurred. The subjects did not take any ascorbic acid or lactose on the test day. The last dose of vitamin or placebo was taken the night before the test day. The last dose of vitamin or placebo was taken the night before the test day. The day before and after the alcohol test day, blood samples were taken and the behavioral tests were performed to establish baseline values.

Test day. The test day began at $8 \mathrm{AM}$ with the insertion of an indwelling catheter into a forearm vein, and a single blood sample was taken. A small breakfast was served at 8:15 $\mathrm{AM}$ and at $9 \mathrm{AM}$ alcohol consumption began. Each subject consumed a total of 0.95 gm ethanol $/ \mathrm{kg}$ body weight. The dose was divided into five equal amounts diluted to a $30 \%$ solution in gingerale and consumed during a $2 \frac{1}{2} 2$-hour period. Blood samples were drawn every hour from $10 \mathrm{AM}$ to 7 PM for determination of the biochemical parameters. Breath samples for the determination of blood ethanol concentration were taken every $1 / 2$ hour from 9:30 AM until ethanol was cleared from the blood. Breath samples were analyzed with the Alco-Sensor III manufactured by Intoximeters, Inc., St. Louis, Mo. Chemical analysis of the blood by a GC head-space gas method was used to ensure the accuracy of the breath analyzer. By 11:30 AM the alcohol was consumed and by 12 noon the blood ethanol concentration had reached a maximum level. Behavioral tests to measure motor coordination, intellectual function, and color discrimination were performed between 12 noon and 12:30 PM. Lunch was served at 12:45 PM and dinner at 5:30 PM. The test day ended at 7 PM.

Blood analyses. Serum was isolated by centrifugation of whole blood 30 minutes after it was drawn. Serum ascorbic acid levels were measured by the HPLC method of Sauberlich et al. ${ }^{12}$

Lactate levels were determined in whole blood by the enzymatic, fluorometric method of Passonneau. ${ }^{13}$ Pyruvate levels were determined in whole blood by the enzymatic, fluorometric method of Passonneau and Lowry. ${ }^{14}$

SGOT and SGPT levels were determined by the spectrophotometric method of Kessler et al. ${ }^{15}$ Levels of serum $\gamma$-glutamyltranspeptidase (SGGT) were determined by the spectrophotometric method of Szasz. ${ }^{16}$ 


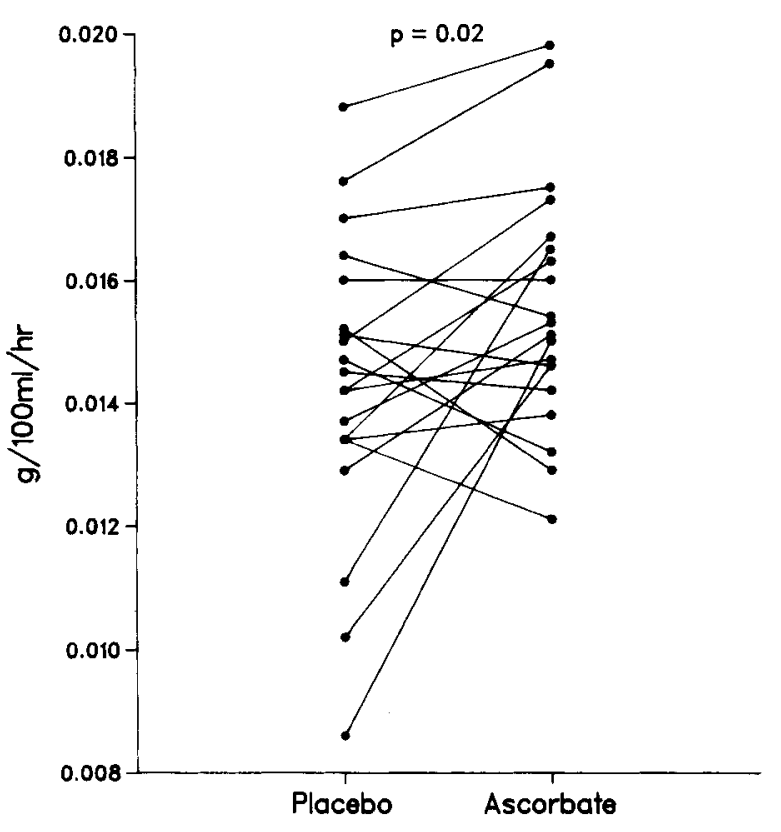

Fig. 1. Effect of ascorbic acid pretreatment on blood ethanol clearance after an acute dose of $0.95 \mathrm{gm}$ ethanol $/ \mathrm{kg}$ body weight in 20 subjects.

Serum triglyceride levels were determined by the enzymatic, spectrophotometric method of Bucolo and David. ${ }^{17}$

Behavioral tests. All behavioral tests were administered and scored by the same individual who was unaware of the pretreatment regimen. Before the first baseline value was obtained, each subject performed the tests a number of times to become familiar with them and achieve a consistent score. During the actual conduct of the study, all tests were run in duplicate and the values expressed as the mean except for the color discrimination test, which was administered singly.

Motor coordination was assessed with the use of the established "Finger-Finger" test developed by Goldberg. ${ }^{18} \mathrm{~A}$ paper-covered disk, $3 \frac{1}{2} 2$ inches in diameter, was attached to one forefinger of the subject and a thimble with a pointed steel tip was attached to the other forefinger. The subject (with arms extended) was asked to bring the forefingers together repeatedly, producing a pattern of dots on the paper. The area enclosed by the dots was used as the index of motor coordination. The area enclosed by the dots was determined with the use of an Apple IIE computer.

Intellectual function was assessed with the use of the "Serial Sevens" test established by Goldberg. ${ }^{18}$ The subject subtracted 7 successively from a number between 99 and 104. Numeric measures of speed and accuracy were recorded.

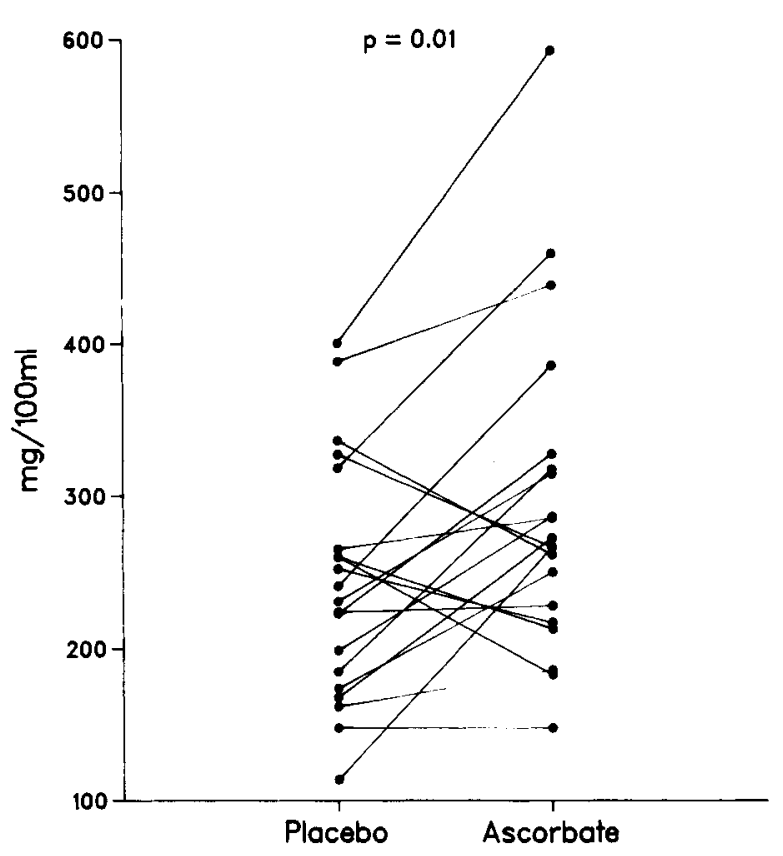

Fig. 2. Effect of ascorbic acid pretreatment on serum triglyceride levels after an acute dose of $0.95 \mathrm{gm}$ ethanol $/ \mathrm{kg}$ body weight in 20 subjects.

The Farnsworth-Munsell 100 Hue Color Test was used for the measurement of color discrimination. ${ }^{11}$ The test consists of 85 separate plastic caps of finely varied hues that the observer arranges in order according to hue.

Data analysis. Values for serum triglycerides, serum enzymes, and blood lactate-pyruvate ratios were plotted against time. Consistent baseline values were obtained. For each biochemical parameter, the baseline values were uninfluenced by the pretreatment regimen; there was no statistically significant difference in baseline values after either pretreatment regimen. The peak values obtained on the test day were used for statistical comparison. Statistical analysis demonstrated that baseline values for the behavioral tests were also uninfluenced by the pretreatment regimen with the exception of the color discrimination test, which varied $10 \%$ to $50 \%$ between pretreatment regimens. Values obtained on the test day (day of alcohol administration) for motor coordination and intellectual function were used and values obtained on the test day for color discrimination were used after the baseline values were subtracted. Blood ethanol concentration was plotted against time and clearance was calculated by linear regression analysis of the descending slope. The linear portion of the slope after 2 PM was used.

Statistical significance was determined with the twosided, paired Student $t$ test by comparing values ob- 


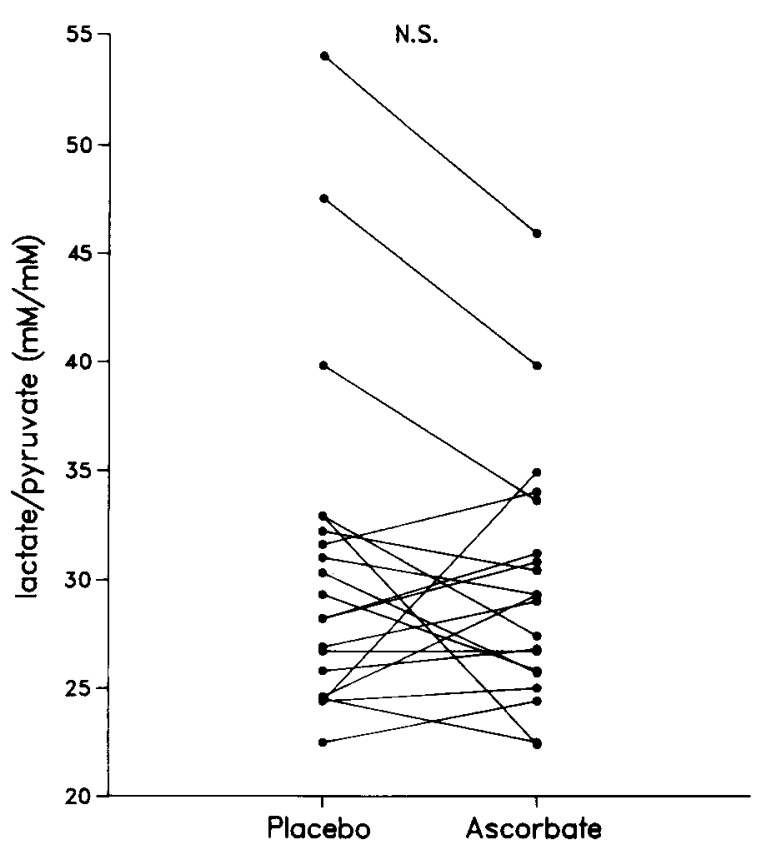

Fig. 3. Effect of ascorbic acid pretreatment on blood lactate/ pyruvate ratio after an acute dose of $0.95 \mathrm{gm}$ ethanol $/ \mathrm{kg}$ body weight in 20 subjects.

tained after ascorbic acid pretreatment with values obtained after placebo pretreatment unless specified otherwise. Differences were considered significant at a $P$ value $<0.05$.

\section{RESULTS}

Ethanol was absorbed rapidly into the blood, with blood ethanol concentrations rising steadily during the period of consumption. For all subjects, irrespective of pretreatment regimen, maximum blood ethanol levels were observed with the breath sample taken at 12 noon, 30 minutes after cessation of drinking. There was no statistically significant difference in the maximum blood ethanol level whether the subject was pretreated with placebo or the vitamin. The mean maximum blood ethanol concentration was $0.074 \pm 0.009 \mathrm{gm} / \mathrm{dl}$ after placebo pretreatment and $0.078 \pm 0.010 \mathrm{gm} / \mathrm{dl}$ after vitamin pretreatment. It is important to note that it was when the blood ethanol concentration was at its highest and not statistically different after either pretreatment regimen that the behavioral tests were administered.

However, ascorbic acid pretreatment did result in a significant increase in blood ethanol clearance $(\mathrm{P}=$ 0.02 ; Fig. 1). The $P$ value represents statistical analysis of all of the data points. Ten subjects demonstrated $>10 \%$ difference in clearance after vitamin pretreatment as compared with placebo pretreatment. Nine of

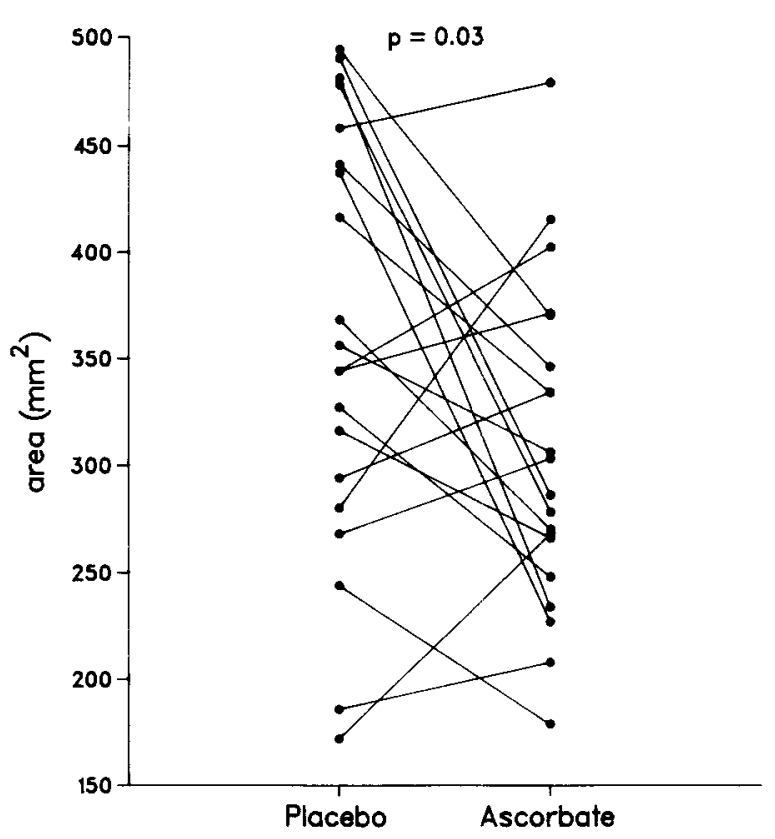

Fig. 4. Effect of ascorbic acid pretreatment on motor coordination after an acute dose of $0.95 \mathrm{gm}$ ethanol $/ \mathrm{kg}$ body weight in 20 subjects.

these 10 subjects had an $11 \%$ to $74 \%$ increase in clearance; one subject had a $15 \%$ decrease. Of the remaining 10 subjects, four had an increase in clearance from $1 \%$ to $10 \%$, five had a decrease from $1 \%$ to $10 \%$, and one had no change at all. It is interesting to note that those individuals who had the largest increase in clearance with ascorbic acid pretreatment were those who had the slowest clearances with the placebo. In addition, the individual with the highest increase (74\%) was Oriental . Individuals with this genetic background have an increased probability of possessing atypical forms of alcohol and acetaldehyde dehydrogenases, which may be responsible for altered metabolism and reaction to ethanol. ${ }^{19}$

Ethanol consumption resulted in a twofold to fourfold increase in serum triglyceride levels. However, when ascorbic acid pretreatment is compared with placebo pretreatment, significantly higher levels of serum triglycerides are found ( $P=0.01$; Fig. 2). This $P$ value, and all subsequent $P$ values, represent statistical analysis of all of the data points. Eleven subjects demonstrated $>20 \%$ difference in serum triglyceride levels after ascorbic acid pretreatment as compared with placebo pretreatment. Ten of these 11 subjects had a $36 \%$ to $133 \%$ increase in triglyceride levels; one subject had a $30 \%$ decrease. Of the remaining nine subjects, four had a $10 \%$ to $20 \%$ increase in serum triglyceride levels, 


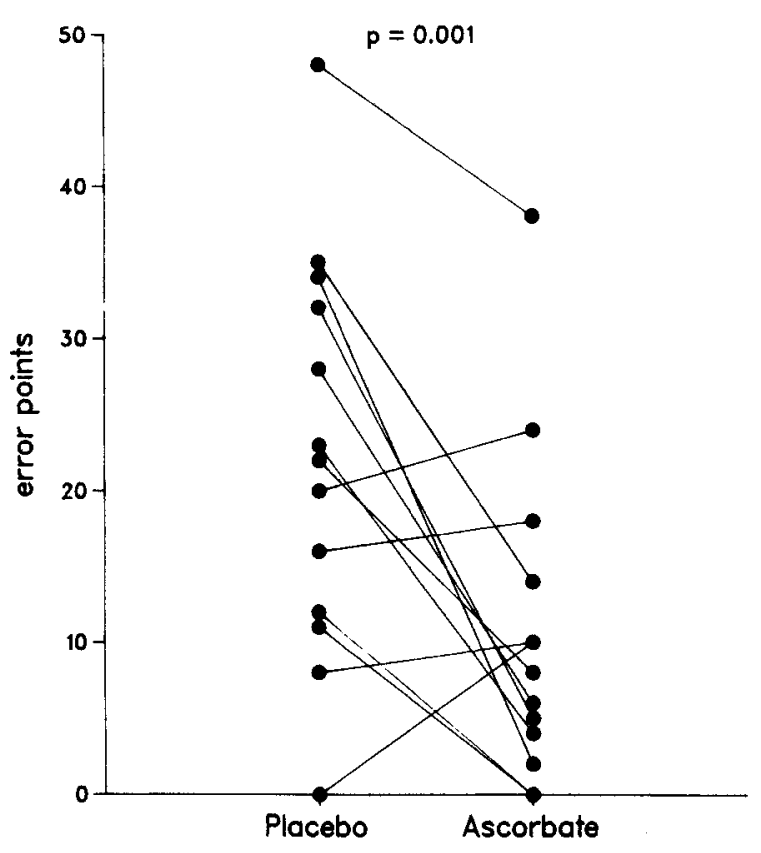

Fig. 5. Effect of ascorbic acid pretreatment on color discrimination after an acute dose of $0.95 \mathrm{gm}$ ethanol $/ \mathrm{kg}$ body weight in 13 subjects.

four had a $1 \%$ to $20 \%$ decrease, and one had no change at all.

Ethanol consumption also resulted in a twofold to fourfold increase in blood lactate/pyruvate ratios. However, no significant difference was found in the ratios when ascorbic acid pretreatment was compared with placebo pretreatment (Fig. 3). Ethanol consumption did not elevate levels of SGOT, SGPT, or SGGT.

Ethanol consumption resulted in impairment of motor coordination. Pretreatment with the vitamin resulted in significantly less impairment $(P=0.03$; Fig. 4). Thirteen subjects demonstrated $>20 \%$ difference in motor coordination after ascorbic acid pretreatment as compared with placebo pretreatment. Eleven of these 13 subjects had a $21 \%$ to $50 \%$ improvement in motor coordination after vitamin pretreatment as compared with placebo pretreatment and two subjects had a decrease of $48 \%$ and $56 \%$. Of the remaining seven subjects, one had an improvement in coordination of $14 \%$ and six had a decrease from $1 \%$ to $20 \%$.

Pretreatment with the vitamin also protected against ethanol's impairment of color discrimination $(\mathrm{P}=$ 0.001 ; Fig. 5). Ten subjects demonstrated $>20 \%$ difference in color discrimination after ascorbic acid pretreatment as compared with placebo pretreatment. Nine of these 10 subjects had a $20 \%$ to $100 \%$ improvement

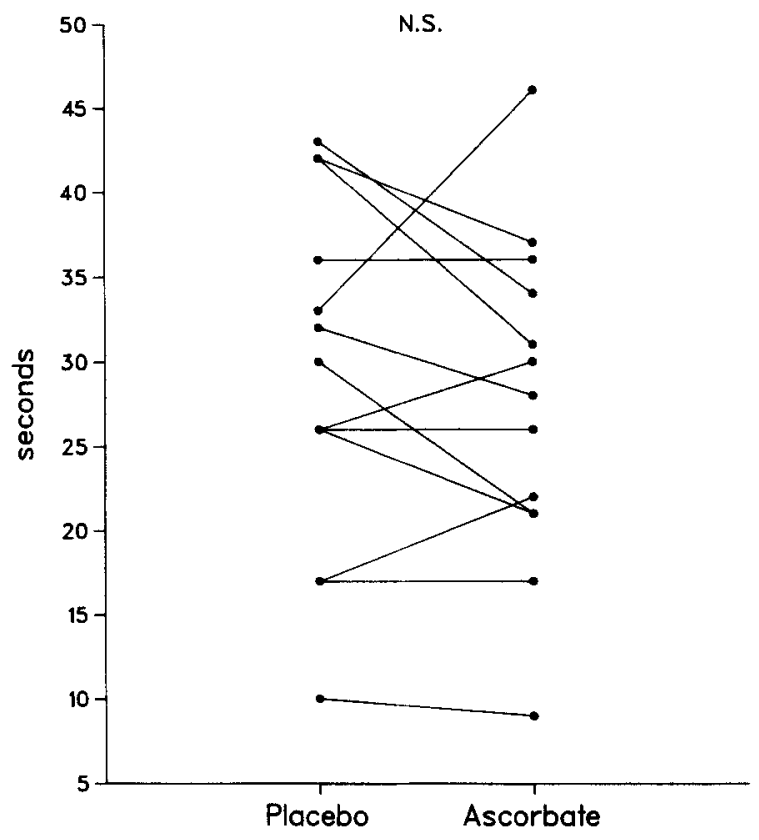

Fig. 6. Effect of ascorbic acid pretreatment on intellectual function after an acute dose of $0.95 \mathrm{gm}$ ethanol $/ \mathrm{kg}$ body weight in 13 subjects.

in color discrimination. The remaining three subjects had a $10 \%$ to $20 \%$ decrease in color discrimination. There are 13 subjects depicted in Fig. 5 because only 13 subjects had impairment in color discrimination after ethanol consumption; the other seven subjects had scores after ethanol consumption that were the same as their baseline values.

Thirteen of the 20 subjects had impaired intellectual function after ethanol consumption as compared with baseline values. However, no statistically significant effect of ascorbic acid pretreatment was observed in these subjects (Fig. 6).

To assess the effect the order of pretreatment had on the results, a statistical comparison with a two-sided Student $t$ test was performed. No statistically significant difference was found between the results of the behavioral tests or the biochemical values and the order in which the pretreatment regimen was administered. In addition, a possible pattern of the individual subject's response across all variables was examined. For example, to determine whether a positive response after vitamin pretreatment in ethanol clearance is associated with an increase in motor performance, elevated triglyceride levels, or enhanced color discrimination, Pearson product-moment correlations were calculated for all possible associations. No statistically significant 
correlation of individual response or lack of response to ascorbic acid pretreatment was found across two or more of the parameters.

Serum ascorbic acid levels were determined at three different time points during the course of the test day. There was no statistically significant difference in the levels of ascorbic acid in the serum during the course of the day nor was there any statistically significant difference in serum ascorbic acid levels for any given subject after the two pretreatment regimens. Mean values of serum ascorbic acid levels after placebo pretreatment were $28 \pm 10 \mu \mathrm{g} / \mathrm{ml}$ and after ascorbic acid pretreatment $28 \pm 11 \mu \mathrm{g} / \mathrm{ml}$. This may be because ascorbic acid is readily secreted from the blood at high doses and serum levels of the vitamin do not accurately reflect tissue levels. ${ }^{20}$ In addition, serum ascorbic acid levels were measured at least 12 hours after cessation of vitamin consumption in a timed-release capsule form.

\section{DISCUSSION}

The metabolism of ethanol is mediated by three enzyme systems. They include alcohol dehydrogenase $(\mathrm{ADH})$, which requires $\mathrm{NAD}^{+}$, catalase, which uses hydrogen peroxide, and the microsomal ethanol-oxidizing system (MEOS), which requires NADPH and oxygen. ADH is considered to be the major pathway in ethanol oxidation in vivo. ${ }^{19}$ MEOS is thought to play a minor role and catalase's contribution is thought to be insignificant since the in vivo rate of hydrogen peroxide generation is limiting. ${ }^{21}$ With regard to the latter, we have previously described and characterized an ascorbic acid-dependent alcohol oxidizing system that is catalyzed by catalase. 'In this system, ascorbic acid generates a peroxide that is used by catalase in a peroxidic oxidation of the alcohol. In the present study the ability of ascorbic acid pretreatment to enhance blood ethanol clearance (Fig. 1) may be the result of its ability to supply peroxide and thus allow catalase to contribute to ethanol oxidation.

It is well established that ethanol affects lipoprotein metabolism and that an acute dose increases levels of serum and hepatic triglycerides. ${ }^{22-24}$ Ethanol causes a number of events that lead to increased hepatic fat levels (e.g., a stress-induced mobilization of fat from adipose tissues, a decrease in the rate of fatty acid oxidation, an increase in the rate of fatty acid synthesis, and an increase in the esterification of fatty acids to triglycerides). In addition, ethanol inhibits export of fat from the liver by impairing the synthesis and secretion of very low-density lipoproteins (VLDL), the export form of triglycerides. The present study demonstrates that with an acute dose of ethanol, ascorbic acid pretreatment causes an increase in serum triglyceride levels (Fig. 2). The possibility that the vitamin increases the rate of fat transport out of the liver should be considered. This could be accomplished by a stimulation in the synthesis or secretion of the VLDL or by preventing alcohol's impairment of these processes. This mechanism would result in less fat accumulation in the liver. In keeping with this, previous in vivo studies in the guinea pig demonstrate that ascorbic acid pretreatment results in less hepatic steatosis after chronic ethanol consumption. ${ }^{2.5}$ Another mechanism to consider involves the stress-induced mobilization of fatty acids from the adipose tissue. The mobilization is mediated via the adrenal gland with release of catecholamines, which causes an increase in adipose tissue triglyceride lipolysis. The adrenal gland contains the highest concentration of the vitamin and it is known that the vitamin is involved in catecholamine metabolism. ${ }^{25,26}$ It is not unreasonable that the adrenal-mediated mobilization of fat may be enhanced by high tissue concentrations of the vitamin. Although elevated serum triglyceride levels could be viewed as a protective effect of ascorbic acid pretreatment if it results in less fat in the liver, it could also be viewed as a detrimental effect if the elevated triglyceride levels proved harmful to other organs.

It is known that increased blood lactate/pyruvate ratios after ethanol consumption reflect the increase in the hepatic NADH/NAD ${ }^{+}$ratio resulting from ethanol oxidation via $\mathrm{ADH}$. Blood lactate/pyruvate levels were monitored to determine if ascorbic acid pretreatment could reduce the hepatic NADH/NAD ${ }^{+}$ratio by providing a non-ADH pathway for ethanol oxidation. Although ascorbic acid pretreatment did significantly increase blood ethanol clearance, there was no significant decrease in the elevated lactate/pyruvate ratio (Fig. 3). It is possible that the degree of enhanced ethanol clearance was not large enough to observe a significant reduction in the lactate/pyruvate ratio.

The participation of ascorbic acid in neurochemical events is of current interest. Its involvement in $\mathrm{Na}^{+} / \mathrm{K}^{+}$transport and metabolism of neurotransmitters including the synthesis of catecholamines, storage of norepinephrine, and regulation of dopaminergic transmission have been reported. ${ }^{26-28}$ In addition, the vitamin has been implicated in modulating the behavioral effects of antipsychotic drugs by influencing their binding to the dopamine receptor. ${ }^{29}$ With regard to ethanol's impairment on behavior, ascorbic acid has been shown to prevent impaired swimming in mice caused by an intoxicating dose of ethanol. ${ }^{6}$ The protective effect of 
ascorbic acid pretreatment on impaired motor coordination caused by acute ethanol consumption demonstrated in this study (Fig. 4) supports a neurochemical role for the vitaminn. The protective effect is not caused by altered blood ethanol concentrations because ascorbic acid pretreatment had no effect on ethanol concentrations at the time the behavioral tests were administered. The mechanism of the protective action, be it through stabilization of the neuronal membrane, an effect on $\mathrm{Na}^{+} / \mathrm{K}^{+}$transport, an effect on the synthesis, storage, release, or binding of the neurotransmitters, or an as yet unknown mechanism, remains to be determined.

Previous studies have also demonstrated ascorbic acid involvement in eye physiology and protection against cataract formation and inhibition of amino acid transport across the cornea membrane, which is caused by oxidative damage. ${ }^{30-33}$ The vitamin's antioxidant and radical scavenging properties, as well as its potential to serve as an energy source for transport processes in the eye, have been implicated in its ability to afford this protection. In addition, ascorbic acid is actively secreted into ocular tissues and, in certain species, concentrations may be twentyfold greater than in plasma. ${ }^{32}$ Impairment in color discrimination by ethanol has been postulated to be caused by a direct toxic effect of ethanol on the inner retina of the eye. ${ }^{11}$ The protection by ascorbic acid against impaired color discrimination could be the result of its antioxidant properties or involvement in membrane transport. However, it is also possible that the impairment in color discrimination is caused by a general central nervous system effect resulting in lack of concentration and enhanced random errors. In this case the protection found with ascorbic acid pretreatment further supports a neurochemical role for the vitamin.

The present clinical study demonstrates that ascorbic acid pretreatment is capable of influencing both biochemical and behavioral changes caused by an acute dose of ethanol. The influence of ascorbic acid pretreatment, although not observed in every subject, was greatest in those subjects with the largest alcohol impairment of behavior and the slowest rates of ethanol elimination after placebo pretreatment. The results of this clinical study coupled with previous animal studies suggest that ascorbic acid may be of value in protecting against some harmful consequences of alcohol consumption in certain individuals. In addition, the determination of the exact mechanisms by which ascorbic acid is acting may lead to the elucidation of important new biologic roles for the vitamin.
We gratefully acknowledge Dr. Dennis Martin and Linda Annesley of the Biochemistry Core Facility of the Michigan Diabetes Research and Training Center for the lactate and pyruvate determinations and Dr. Irving Fox of the Clinical Research Center where the studies were conducted. We would also like to especially acknowledge Kathleen M. Jarvenpaa, Assistant Head Nurse in the Clinical Research Center, for her excellent organizational and technical assistance.

\section{References}

1. Susick RL Jr, Zannoni VG. Ascorbic acid and alcohol oxidation. Biochem Pharmacol 1984;33:3963-9.

2. Yunice AA, Hsu JM, Fahey A, Henry S. Ethanol-ascorbate interrelationship in acute and chronic alcoholism in the guinea pig. Proc Soc Exp Biol Med 1984;177: 262-71.

3. Krasner N, Moore MR, Dow J, Goldberg A. Ascorbic acid and ethanol metabolism. Lancet 1974;21:693-5.

4. Yunice AA, Lindeman RD. Effect of ascorbic acid and zinc sulfate on ethanol toxicity and metabolism. Proc Soc Exp Biol Med 1977;154:146-50.

5. Susick RL Jr, Abrams GD, Zurawski CA, Zannoni VG. Ascorbic acid and chronic alcohol consumption in the guinea pig. Toxicol Appl Pharmacol 1986;84:329-35.

6. Busnel RG, Lehman AG. Antagonist effect of sodium ascorbate on ethanol-induced changes in swimming of mice. Behav Brain Res 1980;1:351-6.

7. Lieber C. Medical disorders of alcoholism: pathogenesis and treatment. In: Major health problems in internal medicine, vol 22. Philadelphia: WB Saunders Co, 1982.

8. Goldberg MB, Watts C. Serum enzyme changes as evidence of liver reaction to oral alcohol. Gastroenterology 1965;49:256-61.

9. Goldstein DB. Pharmacology of alcohol. New York: Oxford University Press, 1983.

10. Lamb RG, Wood CK, Fallon FJ. The effect of acute and chronic ethanol intake on hepatic glycerolipid biosynthesis in the hamster. J Clin Invest 1979;63:14-20.

11. Russell RM, Carney EA, Feiock K, Garrett M, Karawski $P$. Acute ethanol administration causes transient impairment of blue-yellow color vision. Alcohol Clin Exp Res 1980;4:396-9.

12. Sauberlich HE, Green MD, Omaye ST. Determination of ascorbic acid and dehydroascorbic acid. In: Seib PA, Tolbert BM, eds. Ascorbic acid: chemistry, metabolism, and uses. Washington DC: American Chemical Society, 1982:199-221.

13. Passonneau JV. L-Lactate-fluorometric method. In: Bergmeyer HU, ed. Methods of enzymatic analysis, ed 2, vol 3. New York: Academic Press Inc, 1974: 1468-72.

14. Passonneau JV, Lowry OH. Pyruvate-fluorometric assay. In: Methods of enzymatic analysis, ed 2, vol 3. New York: Academic Press Inc, 1974:1452-6.

15. Kessler G, Morgenstern S, Snyder L, Varady R. Improved three point assays for ALT and AST in serum using the technicon SMAC high speed, computer con- 
trolled biochemical analyzer to eliminate the common errors found in enzyme analysis. Presented at the Ninth International Congress on Clinical Chemistry, Toronto, Canada, 1975.

16. Szasz G. A kinetic photometric method for serum gamma-glutamyltranspeptidase. Clin Chem 1969;15: 112-36.

17. Bucolo G, David H. Quantitative determination of serum triglycerides by use of enzymes. Clin Chem 1973;19: 475-82.

18. Goldberg L. Quantitative studies on alcohol tolerance in man. Acta Physiol Scand 1943;5(suppl):1 1-85.

19. Li T-K. Enzymology of human alcohol metabolism. In: Meister A, ed. Advances in enzymology and related areas of molecular biology, vol 45. New York: John Wiley \& Sons Inc, 1977:427-83.

20. Martin GR. Studies on the tissue distribution of ascorbic acid. In: Burns JJ, ed. Vitamin C, vol 92. New York: Annals of the New York Academy of Sciences, 1961: 141-7.

21. Zahlten RN, Nejtek ME, Jacobson JC. Ethanol metabolism in guinea pig: in vivo ethanol elimination, alcohol dehydrogenase distribution, and subcellular localization of acetaldehyde dehydrogenase in liver. Arch Biochem Biophys 1981;207:371-9.

22. Baraona E, Lieber CS. Effect of ethanol lipid metabolism. J Lipid Res 1979;20:289-315.

23. Hansson P, Nilsson-Ehle P. Acute effects of ethanol and its metabolism on plasma lipids and lipoprotein lipase activity. Ann Nutr Metab 1983;27:328-37.

24. Crouse JR, Grundy SM. Effects of alcohol on plasma lipoproteins and cholesterol and triglyceride metabolism in man. J Lipid Res 1984;25:486-96.
25. Zannoni V, Lynch M, Goldstein S, Sato P. A rapid micromethod for the determination of ascorbic acid in plasma and tissues. Biochem Med 1974;11:41-8.

26. Subramanian N. On the brain ascorbic acid and its importance in metabolism of biogenic amines. Life Sci 1977;20:1479-84.

27. Lohmann W. Structure of ascorbic acid and its biological function, VI. Its importance for $\mathrm{Na}^{+} / \mathrm{K}^{+}$transport. Biophys Struct Mech 1984;10:205-10.

28. Rebec GV, Centore JM, White LK, Alloway KD. Ascorbic acid and the behavioral response to haloperidol: implications for the action of antipsychotic drugs. Science $1985 ; 227: 438-40$.

29. Tolbert LC, Thomas TN, Middaugh LD, Zemp JW. Effect of ascorbic acid on neurochemical, behavioral, and physiological systems mediated by catecholamines. Life Sci 1979;25:2189-95.

30. Nishigari H, Hayashi R, Jung R, Lee W, Maruyama K, Iwatsuru M. Preventive effect of ascorbic acid against glucocorticoid-induced cataract formation of developing chick embryos. Exp Eye Res 1985;40:445-51.

31. Consul BN, Nagpal PN. Quantitative study of the variations in the levels of glutathione and ascorbic acid in human lenses with senile cataract. Eye Ear Nose Throat Monogr 1968;47:77-80.

32. Cooperstein DF, Scott WN. Effects of ascorbate and ATP upon amino acid transport in the toad's cornea. Invest Ophthalmol Vis Sci 1978; 17:370-3.

33. Varma SD, Kumar S, Richards RD. Light-induced damage to ocular lens cation pump: prevention by vitamin C. 1979;76:3504-6. 